\title{
63. Note sur le théorème fondamental dans la géométrie conforme des sous-spaces riemanniens.
}

\author{
Par Kentaro Yano et Yosio MuTô. \\ Institut Mathématique, Université Impériale de Tokyo. \\ (Comm. by S. KakeyA, M. 1. A., Dec. 12, 1946.)
}

§0. Dans un Mémoire précédent, ${ }^{(1)}$ nous avons traité le théorème fondamental dans la géométrie conforme des sous-espaces riemanniens, et obtenu cinq équations tensorielles conformes comme la condition nécessaire et suffisante pour que trois tenseurs donnés déterminent un sous-espace plongé dans un espace euclidien el dont les tenseurs fondamentaux conformes sont précisément les tenseurs donnés. Nous allons, dans cette Note, montrer que les dcux de ces cinq équations peuvent être déduites des trois autres.

$\S 1$. Considérons un sous-espace $V_{m}$ à $m$ dimensions

$$
x^{\lambda}=x^{\lambda}\left(u^{i}\right)^{(2)}
$$

plongé dans un espace riemannien à $n$ dimensions, dont le tenseur métrique est $g_{\mu \nu}$. Alors le premier tenseur fondamental $g_{j k}$ du sous-espace est donné par où $B_{j}^{\cdot \lambda}=\frac{\partial x^{\lambda}}{\partial u^{j}}$ désignent $m$ vecteurs, linéairement indejpendents et tangents au sous-espace.

En lésignant par $B_{P}^{\cdot \lambda} n-m$ vecteurs unitaires, normaux au sous-espace et orthogonaux les uns aux autres, on a

$$
g_{\mu \nu} B_{j}^{\cdot \mu} B_{P}^{\cdot \nu}=0 \text { et } g_{\mu \nu} B_{P}^{\cdot \mu} B_{Q}^{\cdot \nu}=\hat{o}_{P Q} \text {. }
$$

Cela étant, les équations de Gauss pour le $V_{m}$ peuvent s'écrire

$$
\left.B_{j ; k}^{\cdot \lambda}=B_{j, k}^{\cdot \lambda}+B_{j}^{\cdot \mu} B_{k}^{\cdot \nu}\left\{\begin{array}{l}
i \\
j k
\end{array}\right\}-B_{i}^{\cdot \lambda}{ }_{j k}^{i}\right\}=H_{j k P} B_{F}^{\cdot \lambda}
$$

où le point-virgule désigne la dérivée covariante le long du souse-space set la virgule la dérivée partielle par rapport à $u^{k},\left\{\begin{array}{c}\lambda \\ \mu \nu\end{array}\right\}$ et $\left\{\begin{array}{l}i \\ j k\end{array}\right\}$ étant les symboles de Christoffel pour $V_{n}$ et $V_{m}$ respectivement. Les $H_{j k P}=H_{k j P}$ apparaissant dans les equations de Gauss sont les seconds tenseurs fondamentaux du sousespace.

D'autre part, les équations de Weingarten peuvent s'écrire

(1) K. Yano et Y. Mutô: Sur le théorème fondamental dans la géométrie conforme des sous-espaces riemanniens. Proc. Physico-Math. Soc. Japan, 24 (1942), 437-449.

(2) Les indices $\left\{\begin{array}{l}i, \mu, \nu, \ldots, \text { prennent respectivement les symboles } \\ i, k, j, \ldots, \\ P, Q, k, \ldots\end{array}\left\{\begin{array}{l}1,2, \ldots, n, \\ \dot{1}, \dot{2}, \ldots, \dot{m}, \\ \dot{m}+\dot{1}, \ldots, n \text {. }\end{array}\right.\right.$ 
No. 11.] Note sur le théorème fondamental dans la géométrie conforme.

$$
B \vec{P} ; k_{i}^{\lambda} \equiv B_{P, k}^{\lambda}+B_{P}^{\cdot \mu} B_{k_{k}^{\nu}}^{\cdot \nu}\left\{{ }_{\mu \nu}^{\lambda}\right\}=-B_{i}^{\cdot \lambda} H_{\cdot k P}^{i}+L_{P Q k} B_{Q}^{\lambda},
$$

où

$$
H_{\cdot k P}^{i}=g^{i j} H_{j k P} \text { et } L_{P Q k}\left(=-L_{Q P k}\right)=g_{\mu \nu} B_{P ; k}^{\cdot \mu} B_{Q}^{\cdot \nu} .
$$

Or, si l'on effectue une transformation conforme

$$
g_{\mu \nu}=\rho^{2} g_{\mu \nu}
$$

du tenseur fondamental $g_{\mu \nu}$, ou $o$ est une fonction arbitraire des coordonnees, les trois tenseurs fondamentax $g_{j k}, H_{j k}$ et $L_{P Q k}$ du sous-espace subissent respectivement des transformations suivantes :

$$
g_{i k}=\rho^{2} g_{j k}, \quad H_{j k P}=\rho\left(H_{j k P}-g_{j k} \rho_{\lambda} B \cdot P_{P}^{\cdot \lambda}\right), \quad \bar{L}_{P Q k}=L_{P Q k} . \quad\left(\rho_{\lambda}=\frac{\partial \log \rho}{\partial x^{\lambda}}\right)
$$

Par conséquent, si l'on définit le tenseur $M_{j k P}$ par

on obtient

$$
M_{j k P}=H_{j k P}-\frac{1}{m} g_{j k} g b H_{b c P},
$$

$$
g_{j \underline{k}}=\rho^{2} g_{j k}, \quad \bar{M}_{j k P}=\rho M_{j k P}, \quad L_{P Q k}=L_{P Q k} .
$$

On les appelle les tenseurs fondamentaux conformes du sous-espace. Le tensour $M_{j k P}$ satisfait à la relation $g^{j k} M_{j k P}=0$.

$\S 2$. Or, nous avons dẻmontré, dans le Mèmoire citè dans $§ 0$, qu'ètant donnés un tenseur symétrique $g_{j k}, n-m$ tenseurs symétriques $M_{j k P}$ satisfaisant à $g^{j k} M_{j k P}=0$ et $(n-m)-(n-m-1) / 2$ vecteurs $L_{P Q k}\left(=-L_{Q P k}\right)$, pour qu'ils déterminent un sous-espace à $m$ dimensions, plongé dans un espace euclidien à $n$ dimensions et dont les trois tenseurs fondamentaux conformes sont $\rho^{t} g_{j k}, \rho M_{j k P}$ et $L_{P Q k}$ où $\rho$ est une fonction convenable, il faut et il suffit qu ils satisfassent aux cinq relations

$$
\begin{aligned}
& C_{\cdot j k h}^{i}=M_{j k P} \cdot M_{\cdot h P}^{i}-M_{j h P} M_{\cdot k P}^{i} \\
& \frac{1}{m-2}\left(M_{j a P} M_{\cdot k P}^{a} \delta_{h}^{2}-M_{j a P} M_{\cdot h P}^{a} \delta_{k}^{2}+g_{j k} M_{\cdot a P}^{2} M_{\cdot h P}^{a}-g_{j h} M_{a P}^{i} M_{\cdot k R}^{a}\right) \\
& -\frac{M_{\cdot b P}^{a} M_{\cdot a P}^{b}}{(m-1)(m-2)}\left(g_{j k} \partial_{i h}^{i}-g_{j h} \partial_{k}^{i}\right),
\end{aligned}
$$

$$
\begin{aligned}
& M_{j k P ; h}-M_{j h P ; k}+M_{j k Q} L_{Q P h}-M_{j h Q} L_{Q P k} \\
& +\frac{g_{j k}}{m-1}\left(M_{\cdot h P: a}^{a}+M^{a} \cdot{ }_{h Q} L_{Q P a}\right)-\frac{g_{j h}}{m-\bar{I}}\left(M^{a}{ }_{k P: a}+M_{\cdot k Q}^{a} L_{Q P a}\right)=0,
\end{aligned}
$$

$$
\begin{aligned}
& L_{P Q k ; h} \cdots L_{P Q h ; k}-M_{k a P} M_{\cdot h Q}^{a}+M_{h a P} M_{\cdot k Q}^{a}+L_{P R k} L_{R Q h}-L_{P R h} L_{R Q k}=0, \\
& \frac{1}{m-3} C_{\cdot j k h ; i}^{i}+\left[\frac{\left(M_{j a P} M_{\cdot k P}^{a}\right), h}{m-2}-\frac{g_{j k}\left(M_{\cdot b P}^{a} M_{\cdot a P}^{b}\right) \cdot h}{2(m-1)(m-2)}\right]
\end{aligned}
$$

$$
-\left[\frac{\left(M_{j a P} M_{\cdot h P}^{a}\right)_{; k}}{m-2}-\frac{g_{j h}\left(M_{\cdot b P}^{a} M_{\cdot a P}^{b}\right)_{i k}}{2(m-1)(m-2)}\right]
$$

$=\frac{1}{m-1} M_{j k P}\left(M_{\cdot h P: a}^{a}+M_{\cdot h Q}^{a} L_{Q P a}\right)-\frac{1}{m-1} M_{j h P}\left(M_{\cdot k P ; a}^{a}+M_{\cdot k Q}^{a} L_{Q P a}\right)$

and

$$
\begin{aligned}
& \frac{1}{m-1}\left(M_{\cdot k P ; a}^{a}+M_{\cdot k Q}^{a} L_{Q P a}\right) ; h-\frac{1}{m-1}\left(M_{\cdot h P ; a}^{a}+M_{\cdot h Q}^{a} L_{Q P a}\right)_{; k} \\
+ & \frac{1}{m-1}\left(M_{\cdot k Q ; a}^{a}+M_{\cdot k R}^{a} L_{R Q a}\right) L_{Q P h}-\frac{1}{m-1}\left(M_{\cdot h Q ; a}^{a}+M_{\cdot h R}^{a} L_{R Q a}\right) L_{Q P R}
\end{aligned}
$$




$$
\begin{aligned}
-\frac{1}{m-2}\left(R_{i k} M_{\cdot h P}^{i}+M_{i a Q} M_{\cdot k Q}^{a} M_{\cdot h P}^{i}-R_{i h} M_{\cdot k P}^{i}\right. & \\
& \left.\quad-M_{i a Q} M_{\cdot h Q}^{a} M_{\cdot k P}^{i}\right)=0 .
\end{aligned}
$$

§. Si l'on pose

$$
C_{j k}=\frac{M_{j a P} M_{\cdot k P}^{a}}{m-2}-\frac{g_{j k} M_{\cdot b P}^{a} M_{\cdot a P}^{b}}{2(m-1)(m-2)}
$$

et

$$
C_{k P}=\frac{1}{m-1}\left(M_{\cdot k P ; a}^{a}+M_{\cdot k Q}^{a} L_{Q P a}\right),
$$

les équations (2.1) et (2.2) peuvent s'écrire respectivement

$$
\begin{aligned}
C_{\cdot j k h}^{i}= & M_{j k P} M_{\cdot h P}^{i}-M_{j h P} M_{\cdot k P}^{i} \\
& +C_{j k} \delta_{h}^{i}-C_{j h} \dot{o}_{k}^{i}+g_{j k} C_{\cdot h}^{i}-g_{j h} C_{\cdot k}^{i}
\end{aligned}
$$

et

$$
\begin{gathered}
M_{j k P ; h}-M_{j h P ; k}+M_{j k Q} L_{Q P h}-M_{j h Q} L_{Q P k} \\
+g_{j k} C_{h P}-g_{j h} C_{k P}=0,
\end{gathered}
$$

où $C_{\cdot k}^{i}=g^{i j} C_{j k}$.

Prenant la dérivée covariante de $M_{j k P} M_{\cdot h P}^{i}-M_{j h P} M_{\cdot k P}^{i}$ et contractant, nous avons

$$
\begin{aligned}
& \left(M_{j k P} M_{\cdot h P}^{i}-M_{j h P} M_{\cdot k P}^{i}\right)_{;} i \\
& =M_{j k P ; i} M_{\cdot h P}^{i}+M_{j k P} M_{\cdot h P ; i}^{i}-M_{j h P ; i} M_{\cdot k P}^{i}-M_{j h P} M_{\cdot k P ; i}^{i} \\
& =M_{j i P ; k} M_{\cdot h P}^{i}+\left(M_{j k P ; i}-M_{j i P ; k}\right) M_{\cdot k P}^{i}+M_{j k P} M_{\cdot h P ; i}^{i} \\
& -M_{j i P ; h} M_{\cdot k P}^{i}-\left(M_{j h P: i}-M_{j i P ; h}\right) M_{\cdot k P}^{i}-M_{j h P} M_{\cdot k P ; i}^{i} \\
& =\left(M_{j i P} M_{\cdot h P}^{i}\right)_{; k}-\left(M_{j i P} M_{\cdot k P}^{i} ; h+M_{j i P}\left(M_{\cdot k P ; h}^{i}-M_{\cdot h P ; k}^{i}\right)\right. \\
& +\left(M_{j k P: i}-M_{j i P ; k}\right) M_{\cdot h P}^{i}-\left(M_{j h P ; i}-M_{j i P ; h}\right) M_{\cdot k P}^{i} \\
& +M_{j k P} M_{\cdot h P ;}^{i}-M_{j h P} M_{\cdot k P: i}^{i}
\end{aligned}
$$

En y substituant (3.4), on trouve

$$
\begin{aligned}
& \left(M_{j k P} M^{i} \cdot h P-M_{j h P} M_{\cdot k P}^{i}\right)_{; i} \\
& =\left(M_{j i P} M_{h P_{j}}^{i}\right)_{k}-\left(M_{j i P} M{ }_{k p P}^{i}\right)_{i} h \\
& -M_{j i P}\left(M_{k Q}^{i} L_{Q P h}-M_{\cdot h Q}^{i} L_{Q P k}+\delta_{k}^{i} C_{h P}-\delta_{h}^{i} C_{k P}\right) \\
& -\left(M_{j k Q} L_{Q F i}-M_{j i Q} L_{Q F k}+g_{j k} C_{i P}-g_{j i} C_{k P}\right) M_{\cdot h P}^{i} \\
& +\left(M_{j h Q} L_{Q P i}-M_{j i Q} L_{Q P h}+g_{j h} C_{i P}-g_{j i} C_{h P}\right) M^{i} \cdot k P \\
& \text { d'où } \\
& +M_{j k P} M_{\cdot h P ; i}^{i}-M_{j h P} M^{i} . k P ; i,
\end{aligned}
$$

(3.5) $\left(M_{j k P} M_{\cdot h P}^{i}-M_{j h P} M_{\cdot k P}^{i}\right)_{;} i$

$=\left(M_{j i P} M_{\cdot h P}^{i}\right)_{; k}-\left(M_{j i P} M_{k p P}^{i} ; h\right.$

$-(m-3)\left(M_{j k P} C_{h P}-M_{j h P} C_{k P}\right)-g_{j k} M^{i} \cdot h P C_{i P}+g_{j h} M_{\cdot k P}^{i} C_{i P}$.

D'autre part, en dérivant $C_{\cdot k}^{i}$ covariantement et contractant, nous avons

$$
\begin{aligned}
C_{\cdot k ; i}^{i} & =\frac{1}{m-2}\left(M_{\cdot a P ; i}^{i} M_{\cdot k P}^{a}+M_{\cdot a P}^{i} M_{\cdot k P ; i}^{a}\right)-\frac{\left(M_{\cdot b P}^{a} M_{\cdot a P}^{b}\right)_{; k}}{2(m-1)(m-2)} \\
& =\frac{1}{m-2}\left(M_{\cdot a P ; i}^{i} M_{\cdot k P}^{a}+M_{a P}^{i} M_{\cdot i P: k}^{a}\right)
\end{aligned}
$$


No. 11.] Note sur le théorème fondamental dans la géométrie conforme.

$$
+\frac{1}{m-2} M_{\cdot a P}^{i}\left(M_{\cdot k P ; a}^{a}-M_{\cdot i P ; k}^{a}\right)-\frac{\left(M_{\cdot b P}^{a} M_{\cdot a P}^{b}\right) ; h}{2(m-1)(m-2)}
$$

En y substituant (3.5), on trouve

$$
\begin{gathered}
C_{\cdot k ; i}^{i}=\frac{1}{m-2}\left(M_{\cdot a P ; i}^{i} M_{\cdot k P}^{a}+M_{a P}^{i} M_{\cdot i P ; k}^{a}\right) \\
-\frac{1}{m-2} M_{\cdot a P}^{i}\left(M_{\cdot k Q}^{a} L_{Q P i}-M_{\cdot i Q}^{a} L_{Q P k}+\delta_{k}^{a} C_{i P}-\delta_{i}^{a} C_{k P}\right) \\
-\frac{\left(M_{\cdot b P}^{a} M_{\cdot a P}^{b}\right) ; k}{2(m-1)(m-2)},
\end{gathered}
$$

d'où

$$
C_{\cdot k ; i}^{i}=M_{\cdot k P}^{a} C_{a P}+\frac{\left(M_{\cdot b P}^{a} M_{\cdot a P}^{b}\right) ; k}{2(m-1)}
$$

Les $\left(M_{j k P} M_{\cdot h P}^{i}-M_{j h P} M_{\cdot k P}^{i}\right)_{; i}$ et $C_{\cdot k ; i}^{i}$ étant ainsi calculés, nous allons dé river (3.3) covariantement et contracter, alors, on aura

$$
\begin{aligned}
C_{\cdot j k h: i}^{i}=\left(M_{j k P} M_{\cdot h P}^{i}-\right. & \left.M_{j h P} M_{\cdot k P}^{i}\right)_{: i} \\
& +C_{j k ; h}-C_{j h ; k}+g_{j k} C_{\cdot h ; i}^{i}-g_{j h} C_{\cdot k ; i}^{i} .
\end{aligned}
$$

En substituant, dans cette équation, (3.5) et (3.6), on trouve

$$
\begin{aligned}
& C_{\cdot j k h ; i}^{i}=-(m-3)\left(C_{j k ; h}-C_{j h ; k}\right) \\
&-(m-3)\left(M_{j k P} C_{h P}-M_{j \dot{h} P} C_{k P}\right) .
\end{aligned}
$$

Cette équations n'est autre que l'équation (2.4). Donc, l'équation (2.4) peut être déduite des équations (2.1) et (2.2).

$\S 4$. En prenant la dérivée covariante de

$$
M_{\cdot k P ; h}^{i}-M_{\cdot h P ; k}^{i}+M_{\cdot k Q}^{i} L_{Q P h}-M_{\cdot h Q}^{i} L_{Q P k}+\delta_{k}^{i} C_{h P}-\delta_{h}^{i} C_{k P}=0
$$

qui est équivalente à (3.4), et en contractant, on obtient

(4.1) $\left(M_{\cdot k P ; h}^{i}-M_{\cdot h P ; k}^{i}\right)_{; i}+\left(M_{k Q}^{i} L_{Q P h}-M^{i} \cdot h Q L_{Q P k}\right)_{i}+C_{h P ; k}-C_{k P ;}=0$.

Or, nous avons

$$
\begin{aligned}
& \left(M_{\cdot k P ; h}^{i}-M_{\cdot h P ; k}^{i}\right)_{i} i \\
& =M_{k P ; h ; i}^{i}-M_{\cdot h P ; k ; i}^{i} \\
& =M_{\cdot k P ; i ; h}^{i}+\left(M_{\cdot k P ;}^{i}, i-M_{\cdot k P ; i}^{i} h\right) \\
& \text { - } M_{\cdot h P ; i ; k}^{i}-\left(M_{\cdot h P ; k ; i}^{i}-M_{\cdot h P ; i ; k}^{i}\right) \\
& =M_{\cdot k P_{i} i, h}^{i}+\left(M_{\cdot k P}^{a} R_{\cdot a h i}^{i}-M_{\cdot a P}^{i} R_{\cdot k h i}^{a}\right) \\
& -M_{\cdot h P ; i ; k}^{i}-\left(M_{\cdot h P}^{a} R_{\cdot a k i}^{i}-M_{\cdot a P}^{i} R_{\cdot h k i}^{a}\right) \text {, }
\end{aligned}
$$

d'où

(42) $\left(M_{\cdot k P ; h}^{i}-M_{\cdot h P ; k}^{i}\right) ; i$

$$
=M_{\cdot k P ; i ; h}^{i}-M_{\cdot h P ; i ; k}^{i}+M_{\cdot k P}^{a} R_{a h}-M_{\cdot h P}^{a} R_{a k},
$$

en vertu de l'identité

$$
M_{a P}^{i} R_{\cdot k h i}^{a}=M_{a P}^{i} R_{\cdot h k i}^{a}
$$

D'autre part, on a

$$
\begin{aligned}
& \left(M_{k Q}^{i} L_{Q P h}-M_{h Q}^{i} L_{Q P k}\right)_{;} i \\
= & M_{k Q ; i}^{i} L_{Q P h}+M_{\cdot k Q}^{i} L_{Q P h ; i}-M_{\cdot h Q ; i}^{i} L_{Q P k}-M_{h Q}^{i} L_{Q P k ; i}
\end{aligned}
$$




$$
\begin{aligned}
= & M_{k Q ; i}^{i} L_{Q P h}+M_{k Q}^{i} L_{Q P i ; h}+M_{k Q}^{i}\left(L_{Q P h ; i}-L_{Q P i ; h}\right) \\
& -M_{h Q ; i}^{i} L_{Q P k}-M \cdot h Q L_{Q P ; k}-M_{h Q}^{i}\left(L_{Q P k ; i}-L_{Q P i ; k}\right) .
\end{aligned}
$$

En y substituant (2.3), on trouve

$$
\begin{aligned}
& \left(M_{k Q}^{i} L_{Q P h}-M_{\cdot h Q}^{i} L_{Q P k}\right)_{i} i \\
& =M_{k Q ; i}^{i} L_{Q F h}+M_{\cdot k Q}^{i} L_{Q P i ; h} \\
& +M_{\cdot k Q}^{i}\left(M_{h a Q} M_{\cdot i P}^{a}-M_{i a Q} M_{\cdot h P}^{a}-L_{Q R h} L_{R P i}+L_{Q R i} L_{R P h}\right) \\
& \text { - } M_{h Q ; i}^{i} L_{Q P k}-M^{i} \cdot h Q L_{Q P i ; k} \\
& -M_{\cdot h Q}^{i}\left(M_{k a Q} M_{\cdot i P}^{a}-M_{i a Q} M_{\cdot k P}^{a}-L_{Q R k} L_{R F i}+L_{Q R i} L_{R P k}\right) \\
& =(m-1) C_{k Q} L_{Q P h}+M_{\cdot k Q}^{i} L_{Q P i ; h}-M_{k Q .}^{i} M_{i a Q} M_{\cdot h P}^{a} \\
& \text { - (m-1) } C_{h Q} L_{Q P k}-M_{\cdot h Q}^{i} L_{Q P i}: . k+M_{\cdot h Q}^{i} M_{i a Q} M_{\cdot k P}^{i} \\
& \text { - }\left(M_{\cdot k Q}^{i} L_{Q R h}-M_{\cdot h Q}^{i} L_{Q R k}\right) L_{R P i_{2}}
\end{aligned}
$$

d'où

$$
\begin{aligned}
& \left(M_{k Q}^{i} L_{Q P k}-M_{\cdot h Q}^{i} L_{Q P k}\right)_{; i} \\
= & (m-2) C_{k Q} L_{Q P h}+\left(M_{k Q}^{i} L_{Q P i}\right)_{i h}-M_{k Q}^{i} M_{i a Q} M_{\cdot h P}^{a} \\
- & (m-2) C_{h Q} L_{Q P k}-\left(M_{h Q}^{i} L_{Q P i}\right)_{;}+M_{h Q}^{i} M_{i a Q} M_{\cdot k P .}^{a} .
\end{aligned}
$$

En substituant (4.2) et (4.3) dans (4.1), on obtient

$$
\begin{aligned}
& (m-2)\left(C_{k P ; h}-C_{h P ; k}\right)+(m-2)\left(C_{k Q} L_{Q P h}-C_{h Q} L_{Q P k}\right) \\
+ & \left(M_{\cdot k P}^{a} R_{a h}+M_{i a Q} M_{\cdot k Q}^{a} M^{i} \cdot h P-M_{\cdot h P}^{a} R_{a k}-M_{i a Q} M^{\cdot} \cdot h Q M_{\cdot k P}^{i}\right)=0 .
\end{aligned}
$$

Ce n'est autre que l'équation (2.5). Donc, l'équation (2.5) peut être déduite des équations (2.2) et (2.3).

$\S 5$. Nous avons donc démontré .que:

La condition nécessaire et suffisante pour qu'un tenseur symétrique $g_{j k}, n-m$ tenseurs symetriques $H_{j k P}$ et $(n-m)(n-m-1) / 2$ vecteurs $L_{P Q k}\left(=-L_{Q P k}\right) d \dot{e}$ terminent un sous-espace à $m$ dimensions plongé dans un espace euclidien $\grave{a} n$ dimensions est que les tenseurs fondamentaux satisfassent aux equations (2.1), (2.2) et (23). 